\title{
Rolling-Sliding Laboratory Tests of Friction Modifiers in Leaf Contaminated Wheel-Rail Contacts
}

\author{
Z. Li $\cdot$ O. Arias-Cuevas $\cdot$ R. Lewis · \\ E. A. Gallardo-Hernández
}

Received: 17 July 2008/Accepted: 4 November 2008/Published online: 19 November 2008

(c) The Author(s) 2008. This article is published with open access at Springerlink.com

\begin{abstract}
Leaf-related adhesion problems have been present in many railway networks all over the world in the last few decades. Since the early 1970s many measures have been undertaken in order to mitigate the problem. One of the measures adopted by many railway networks is the use of friction modifiers. However, the low adhesion problem still persists. Furthermore, the effectiveness of these friction modifiers has not well proven yet due to the lack of research in controlled conditions. Consequently, the rolling stock operators and infrastructure managers do not clearly understand the performance and side effects of the friction modifiers used on their networks. In this paper, an investigation of the performance of two existent friction modifiers in controlled laboratory conditions is presented. These friction modifiers have been used or tested in several railway networks. A twin-disk roller rig has been used to study their performance in leaf contaminated contacts. The adhesion characteristics of both friction modifiers are examined for different slip ratios. The constituents of the friction modifiers are identified and the solid components are analyzed. In addition, damage that these friction modifiers may cause to wheel and rail is also discussed.
\end{abstract}

Keywords Railroad · Friction modifiers ·

Rolling-sliding friction - Leaf contamination .

Low wheel-rail adhesion

Z. Li · O. Arias-Cuevas $(\bowtie)$

Faculty of Civil Engineering and Geosciences, Section of Road and Railway Engineering, Delft University of Technology,

Stevinweg 1, 2628 CN Delft, The Netherlands

e-mail: o.ariascuevas@tudelft.nl

R. Lewis · E. A. Gallardo-Hernández

Department of Mechanical Engineering, The University

of Sheffield, Mappin Street, S1 3JD Sheffield, UK

\section{Introduction}

The friction available between wheel and rail during braking and traction operation is known in the railway terminology as adhesion. It is a crucial factor for the railway industry as a minimum level of adhesion is required for an appropriate braking and traction performance of the rail vehicles. Adhesion is influenced by many factors such as vehicle speed, wheel slip, contact pressure, environmental conditions and natural contaminants. The major cause of decreasing adhesion is the natural contamination; water, rust, oil, and leaves, have been identified as being mainly responsible [1-5]. The combination of leaves and a small amount of water has been reported to bring the lowest adhesion levels, as it occurs during the autumn season [6]. The leaves are normally swept up onto the rails by wind or the train's slipstream, where they are crushed under the high wheel-rail contact pressure. Consequently, a Teflon-like leaf layer is formed, which has black coloration and is hard to remove. This layer is known to have low shear strength and high electrical resistance, which may bring about two negative consequences: low adhesion and electrical isolation [6]. When low adhesion occurs, delays in the train service are the clearest consequence to the railway commuters. However, many other negative effects can arise, such as damages to wheels and rails, signals passed at danger, station platform overruns and, even, collisions. Flat wheels and rail burns caused by low adhesion may also lead to rolling contact fatigue defects such as squats [7]. Therefore, not only the punctuality, but also the safety of the passengers can be threatened if low adhesion situations are encountered. Moreover, the annual costs of low adhesion to the railway industry were reported to be $£ 50$ million in United Kingdom [6] and 100 million SEK in Sweden [8]. In The Netherlands, extreme low 
adhesion conditions on a day in the autumn of 2002 caused wheel defects to increase up to $20 \%$ of the railway fleet, forcing the train operator to stop the services on most of the sections of the network during that day [9].

Several studies on wheel-rail adhesion in leafy contacts have already been conducted in both laboratory and field tests. A report on low adhesion published by the Rail Safety and Standards Board (RSSB) gives a good overview of the research and findings on leaf-related adhesion problems in the last decades [5]. Recently, a study into the characteristics of the leaf layer contamination was carried out by AEA Technology Rail (DeltaRail Group) in UK [10]. They used a full scale wheel-on-rail test rig to produce leaf layer samples, whose mechanical and chemical properties were examined. The shear strength of the leaf layer was found to be inversely proportional to the moisture level; the thickness of the generated layer ranged from 10 to $100 \mu \mathrm{m}$, which implies that the metal surface asperities of wheel and rail will not touch each other in the presence of a leaf layer. The samples were analyzed using Fourier Transform Infra-Red (FTIR) spectroscopy; lignin, cellulose, and pectin were found as the major constituents contributing to the adherence of leaf to the rail. Validation of the laboratory test results was undertaken by comparison with those of samples taken from the British railway network. Laboratory tests to investigate the friction behavior in leaf contaminated contacts have also been carried out with pin-on-disk [8], ball-on-disk [11], and twin-disk [12] tribometers. Olofsson and Sundvall [8] showed in their pioneering laboratory work the influence of leaf contamination and humidity on sliding friction. In the presence of leaf contamination, the friction was significantly decreased compared to dry contacts; furthermore, the increase in relative humidity led to a decrease in sliding friction. Cann [11] investigated the friction properties of the leaf layers under different rolling speeds and slip ratios. In her work, she also analyzed the post-test contamination leaf layer using FTIR micro-spectroscopy; pectin and cellulose were found in the samples. She suggests that the water-soluble pectin reacts chemically with the metal to form iron pectate, which causes the black coloration of the leaf layers that has been extensively reported. Gallardo-Hernandez and Lewis [12] obtained the traction curves (up to 5\% slip) in dry and wet leafy contacts. The dry leaf layers gave the lowest adhesion values and the micro-hardness measurements showed that dry leaf layers were harder than wet ones. They also carried out tests with sand and leaves, in which an increase in adhesion using sand was observed. Besides, Lewis and Dwyer-Joyce [13] published a study on the wear caused by sanding.

In order to fight low adhesion, some practical measures have already been applied in the abovementioned countries, such as vegetation management, rail cleaning methods, and friction modifiers (FMs) [5, 6, 14]. Simple sanding from the train or locomotive is used on railway networks world wide to overcome adhesion problems [15]. In countries such as The United Kingdom and The Netherlands, other FMs have been used and tested in field during the last years $[5,14]$. However, the low adhesion problem still persists. Furthermore, the effectiveness of these FMs has not been well proven yet due to the lack of research in controlled conditions. Consequently, the rolling stock operators and infrastructure managers do not clearly understand the performance and side effects of the FMs used on their networks. In this paper, a laboratory study of a widely used FM is presented together with another FM designed for wet wheel-rail contacts due to rainfall.

The aim of this work was to examine the performance of two FMs in leaf contaminated contacts. A study of these FMs in dry and wet contacts has already been carried out [16]. Both FMs are water-based and have been designed to increase the adhesion in their respective target contamination conditions. One of the FMs-referred as FMB in this paper-has extensively been used in the autumn season on the Dutch and British railways networks to overcome adhesion problems, especially due to leaves and small amounts of water. In The Netherlands, FMB is primarily applied train-borne to top of both rails by means of a speed dependent pumping system, which delivers $4 \mathrm{cc} / \mathrm{m}$ per rail. The other FM-referred as FMA hereinafter-has been tested in a train depot in Japan to increase adhesion in the presence of water, and it was considered to be a potential adhesion enhancer for leafy contacts, although improvement may be needed. FMA is to be applied to the top of both rails in a very thin layer. In this work, a twindisk roller rig has been used to simulate the wheel-rail contact in controlled laboratory conditions. The adhesion characteristics of the two FMs have been studied in leaf contaminated contacts for three different slip ratios: $0.5,1$, and $2 \%$. The leaf layers obtained during testing have been analyzed by means of FTIR micro-spectroscopy in order to assess the leaf layer removal. The constituents of the FMs have been examined and their influence on adhesion improvement and disks damage has been discussed.

\section{Test Set-Up}

\subsection{Test Roller Rig}

The rolling-sliding tests were conducted on the SUROS (Sheffield University ROlling Sliding) roller rig, shown in Fig. 1. A detailed description of the roller rig is given in [17]. The test disks were mounted on independent shafts. By means of a hydraulic jack, a controlled contact pressure was achieved during the test. The slip ratio between the 
Fig. 1 Schematic representation of the SUROS roller rig

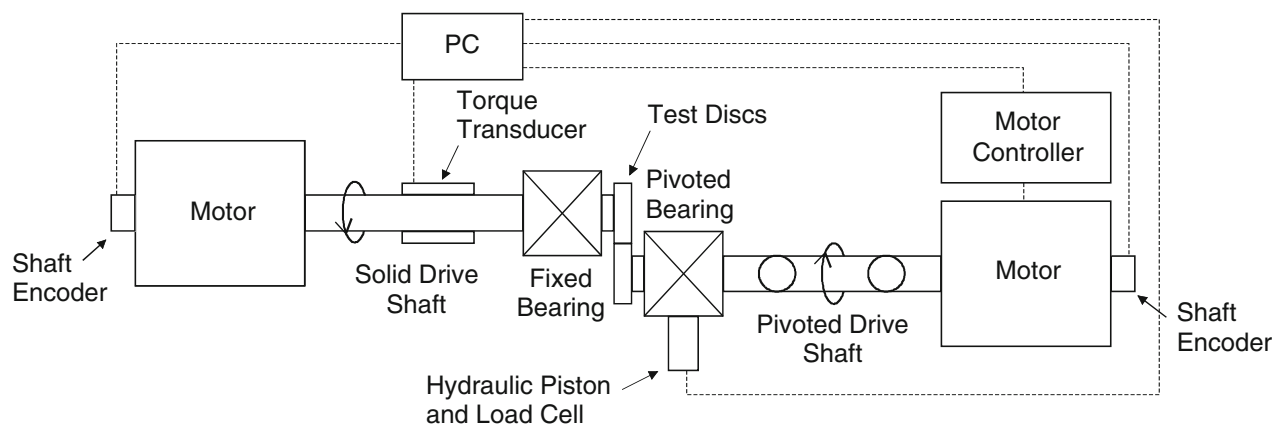

disks was prescribed by setting different rotational speed of the shafts and maintained constant throughout each test with a controller. The slip ratio is defined in Eq. 1, where $w$ and $r$ are the rotational speed and rolling radius of the disks, respectively. The adhesion coefficient was calculated with the readings of the torque transducer and the load cell, as given in Eq. 2 by $T$ and $F_{\mathrm{N}}$, respectively. A personal computer was used to acquire the data and to control both the speed and the load.

$$
\begin{aligned}
& \text { Slip }=\frac{w_{\text {wheel }} \cdot r_{\text {wheel }}-w_{\text {rail }} \cdot r_{\text {rail }}}{w_{\text {wheel }} \cdot r_{\text {wheel }}+w_{\text {rail }} \cdot r_{\text {rail }}} \cdot 200 \% \\
& \mu_{\text {adhesion }}=\frac{T}{F_{\mathrm{N}} \cdot r_{\text {rail }}}
\end{aligned}
$$

\subsection{Test Disks}

The test disks were cut from rails and wheel tires retired from service in the Dutch railway network; R260Mn and B5T steel for the rail and wheel, respectively. The disks were machined with their axes perpendicular to the longitudinal axis of both wheel and rail (see Fig. 2). The Vickers macro-hardness of the wheel and the rail steel used in the tests was measured as $267 \mathrm{HV}_{20 \mathrm{~kg}}$ and $281 \mathrm{HV}_{20 \mathrm{~kg}}$ on average, respectively. Prior to testing, the disks were cleaned in a bath of ethanol by means of ultrasonic vibration. The roughness of the new disks was measured as $1 \pm 0.2 \mu \mathrm{m}$ on average with a profilometer. Before assembling the disks into the roller rig, their diameter was measured with a vernier calliper as necessary for the calculations of slip and adhesion coefficient.

\subsection{Tested Products}

The two water-based FMs tested in this work are applied to the top of the rail in order to increase the wheel-rail adhesion. Microscope photographs of the dried samples are given in Fig. 3. Friction modifier A (FMA) contains several types of solid components, which have different physical and tribological characteristics that provide the final product with varied functionalities such as friction enhancement and film transfer between wheel and rail. Furthermore, there are several polymeric components in FMA, all of which assist in promoting adherence to the wheel and rail steel surfaces. In Fig. 3, it can be seen that the particles agglomerate after drying in an oven.

Friction modifier B (FMB) is a mixture of an inorganic gelling agent, stabilizer, water, sand grains, and stainless steel particles. The gelling agent promotes the adherence of the mix to the wheel and rail surfaces, while the stabilizer provides a reasonable storage life. The stainless steel particles guarantee adequate electrical conductivity of the mix,
Fig. 2 Orientation and dimensions of the disks specimens

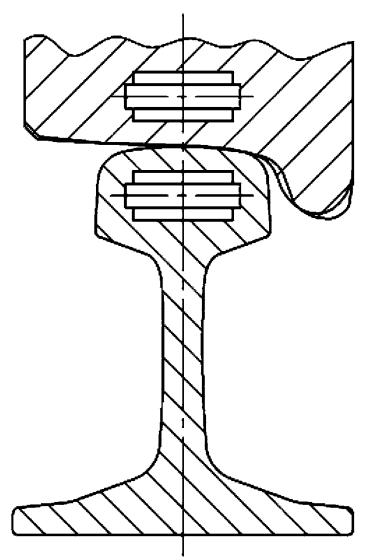

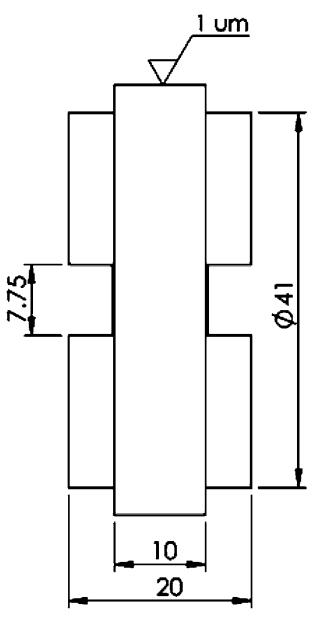


Fig. 3 Microscope photographs of FMA (left) and FMB (right)

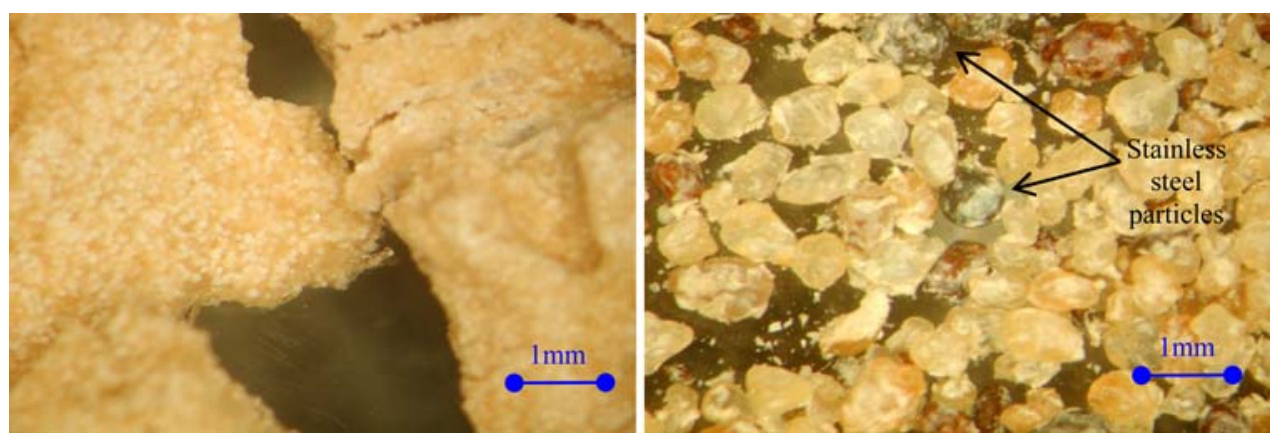

Fig. 4 Dutch sycamore leaves used in the tests (left) and experimental set-up (right)
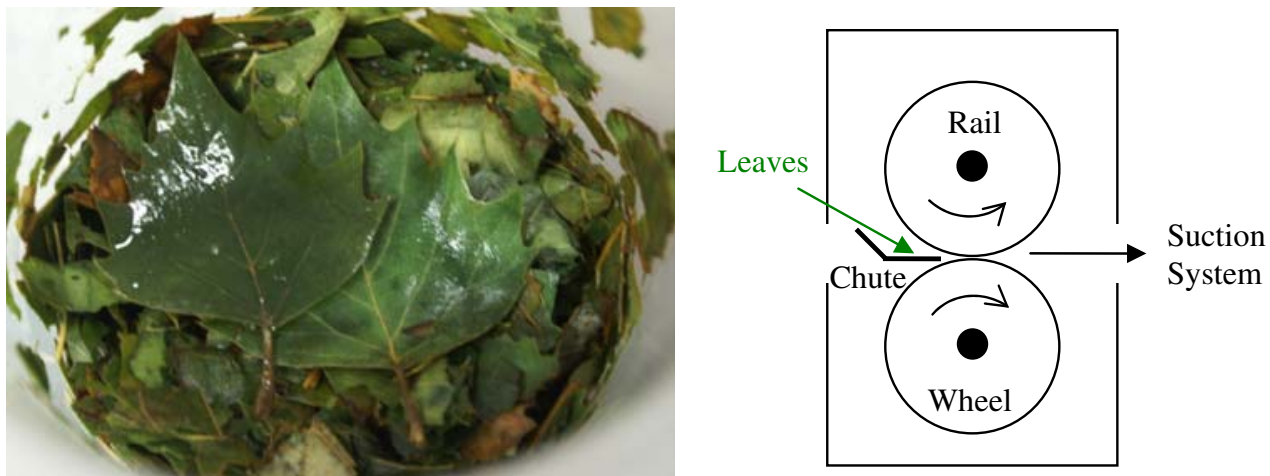

which is necessary for train detection. As FMB is applied from train mounted actuators directly into the wheel/rail contact, the lack of electrically conductive particles could lead to track circuit failure along the network. It can be seen from Fig. 3 that the sand grains vary in size and type, as most probably come from different types of rocks. The black colored particles correspond to the stainless steel, as pointed in Fig. 3.

The leaves used in the experiments were from the sycamore tree. This type of tree is present along the Dutch railroads. The fallen leaves were collected in autumn 2006 in Utrecht, The Netherlands (see Fig. 4). Once they were picked up, they were rinsed in water and frozen at $-80{ }^{\circ} \mathrm{C}$ to preserve their properties for the subsequent testing. Leaves were still partially green, but the petiole was dead. In comparison with fully dead leaves, they should contain more soluble organic compounds.

\subsection{Test Procedure}

In the tests the wheel disk rotated faster than the rail disk; the rotational speed of the rail was maintained at $400 \mathrm{rpm}$, equivalent to $1 \mathrm{~m} / \mathrm{s}$ of rolling speed. Since cylindrical disks were used in the experiments, a line contact of $10 \mathrm{~mm}$ width was present. A load of $4.7 \mathrm{kN}$ was applied on the disks producing a maximum Hertzian pressure of $1.2 \mathrm{GPa}$ in the contact zone, which is representative of that between wheel tread and railhead for passenger trains in The Netherlands. Prior to application, the leaves were defrosted and cut into pieces smaller than the disk contact width to ease their entrapment into the disks interface. The small pieces of leaf were dried out before application. They were manually fed through a chute to the disks interface and being drawn through by a suction system located on the other side of the disks, as depicted in Fig. 4. Initial trials demonstrated that $25 \mathrm{~g}$ of dry leaves were enough to create a relatively hard, durable leaf layer on the disks surface; subsequently, this amount was used for each test. Figure 5 depicts a typical complete test with all the stages. At the beginning of each test, the disks were run for 4,000 cycles at $0.5 \%$ slip to condition the surfaces before the leaves were fed in; then 300-400 cycles were required to apply the necessary amount of leaves. Thus, the leaf layer generation simulated what happens in the real situation, in which repeated wheel passages compact and shear leaves on the top of the rail. Next, the test was stopped to apply the FM and/or to change the slip. The FM was painted onto the rail surface with a brush; care was taken that no leaf layer was removed in this procedure. In Fig. 6, pictures of the disks with leaf layer on their surfaces can be seen as well as the FMs when these were applied. Finally, the test was run for 3,000 cycles to examine the removal of the leaf layer in different contact conditions. For each test conducted with the FMs, a baseline (i.e., no FM applied) was first obtained so as to compare the performance of FMs with the untreated situation. The tests were carried out at $0.5,1$, and $2 \%$ slip, which represent typical values that can be found in the contact between wheel tread and top of the 
Fig. 5 Typical complete adhesion test with run-in, leaf layer formation, and test with leaf layer on disks surface

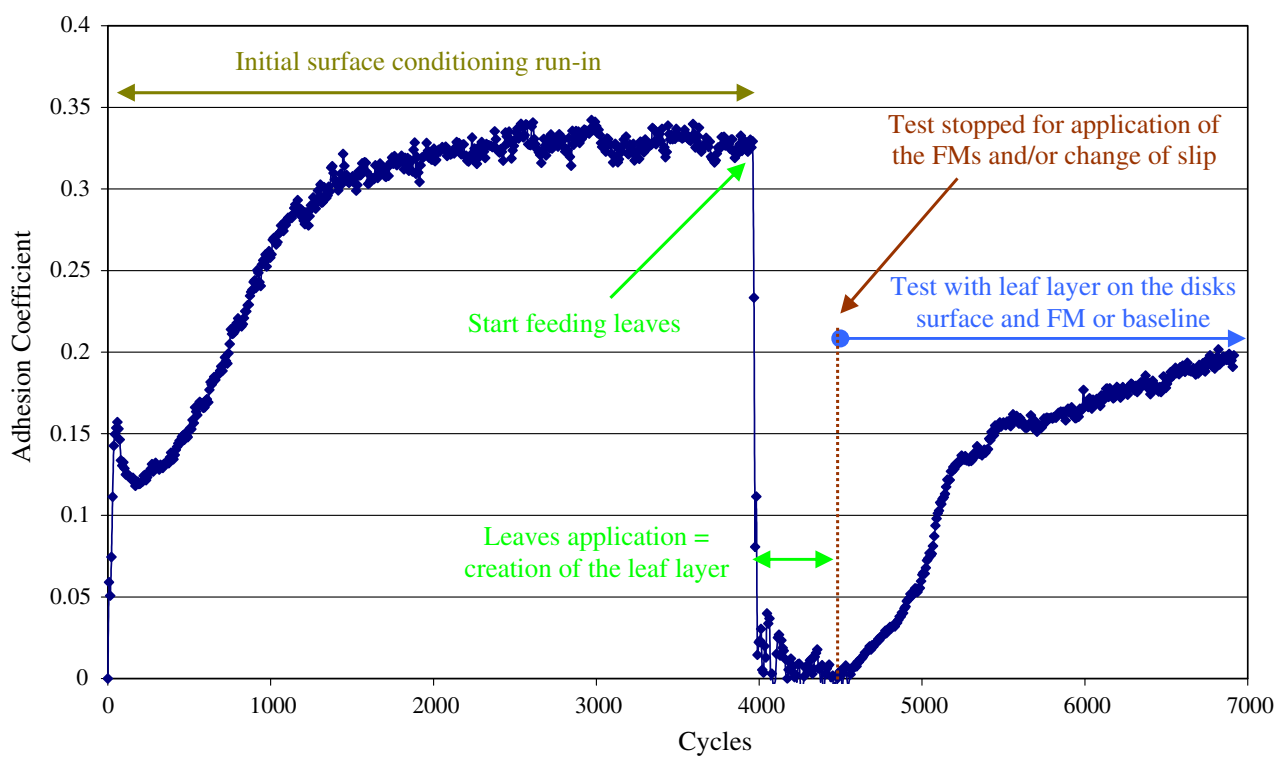

Fig. 6 Disks surfaces after leaf layer has been created and ready to test with: a baseline, b FMA, c FMB
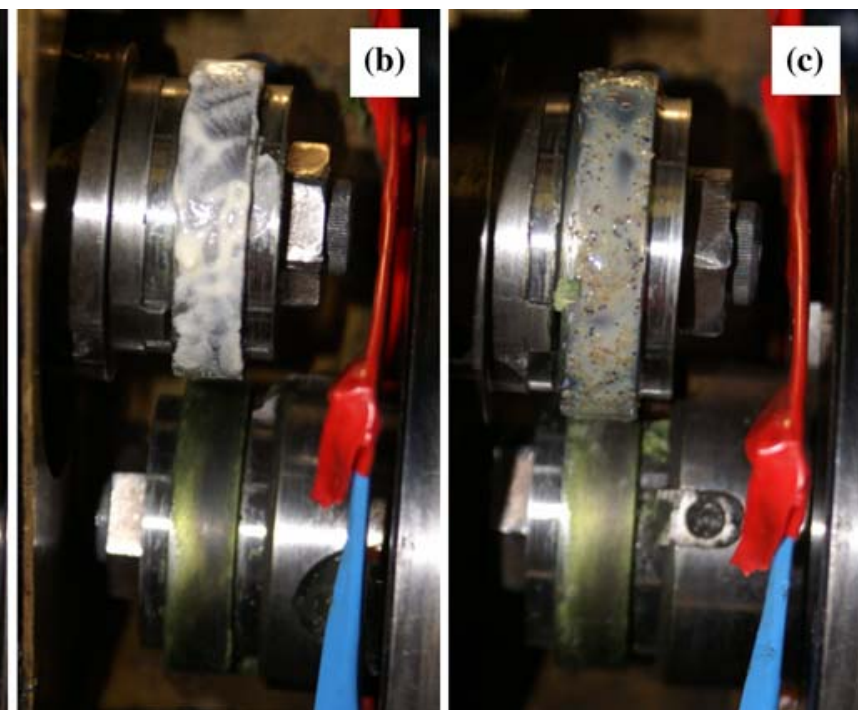

rail. Furthermore, a thermometer and a hygrometer were next to the twin-disk rig. The ambient temperature recorded ranged from $24{ }^{\circ} \mathrm{C}$ to $28{ }^{\circ} \mathrm{C}$ and the relative humidity between $30 \%$ and $45 \%$.

\section{Results}

\subsection{Adhesion Tests}

The adhesion performance of the two FMs and the baseline for a leaf contaminated contacts was investigated. During the feed of leaves, the adhesion coefficients registered were $0.01-0.04$ for $0.5 \%$ slip, which is in agreement with previous work on the same roller rig [12]. Note that on this roller rig an adhesion coefficient of 0.30 is typical of dry uncontaminated contacts for $0.5 \%$ slip $[12,16]$. Once the feed of leaves was stopped and the test started again with the selected slip ratio, the leaf layer was gradually broken and removed from the disks surface with the increasing number of cycles; eventually metal-to-metal contact was reached either partially or completely. The tests were run at $0.5,1$, and 2\% slip, as shown in Figs. 7, 8, and 9; a baseline data for a dry uncontaminated contact is also included (named baseline-dry) for the sake of comparison. In all the tests, FMB showed the best performance in breaking up the layer and, therefore, bringing the adhesion to that of uncontaminated dry contacts. On the contrary, tests with baseline and FMA did not reach the adhesion levels of the baseline-dry contact after 3,000 cycles for the slip ratios considered (see Figs. 7, 8, 9). Moreover, the influence of the leaf layer did not disappear at $0.5 \%$ slip for any of the three cases after 3,000 cycles, indicating that higher slip or more cycles are necessary to deplete the leaf layer completely. However, 
Fig. 7 Leaf contaminated tests at $0.5 \%$ slip (and reference baseline-dry without contamination)

Fig. 8 Leaf contaminated tests at $1 \%$ slip (and reference baseline-dry without contamination)
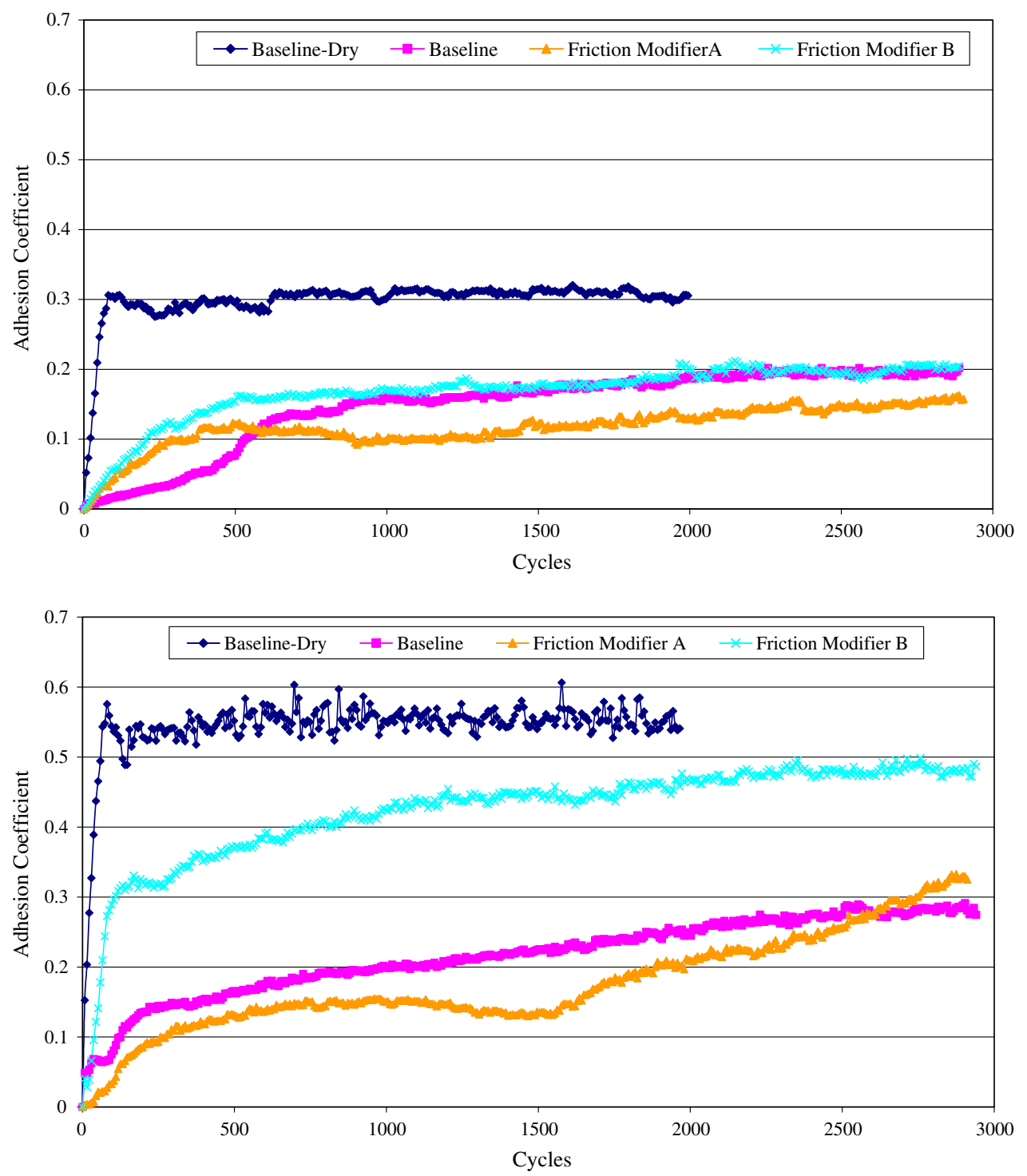

much steeper initial slope was observed in the adhesion curves with both FMs at $0.5 \%$ slip, which led to faster recovery in adhesion compared to the baseline. Furthermore, a moderate adhesion characteristic of FMA was observed in these tests; a third body layer was formed at the disks surface that yields lower adhesion coefficients than the baseline. This moderate adhesion behavior was already observed in a previous work with FMA in dry and wet contacts [16]. In the baseline test at $2 \%$ slip, it seemed that the abrupt increase in slip when the disks are brought into contact could have caused the leaf layer to be depleted, yielding an instantaneous increase of 0.14 in the adhesion coefficient, as indicated in Fig. 9. The abrupt increase in adhesion did not occur for the tests at $2 \%$ with FMs, which may be attributed to their water and solid contents that accommodate the slip.

The level of adhesion necessary for an adequate braking and traction performance depends on the train type, composition, and the traction and braking systems. It is well-known that the adhesion required for braking is lower than for traction. As an example, one of the most adhesion demanding electrical multiple-units running on the Dutch railway network requires 0.14 for braking and 0.24 for traction. In this work, we took as reference the adhesion requirements given in [5], which are 0.09 in braking and 0.2 in traction. Table 1 gives an overview of the number of cycles required for the tests with FMs and the baseline tests to reach those requirements for each slip considered. It can be seen that FMB always reached adequate adhesion levels first for all slip ratios. There seemed to be an optimum slip of $1 \%$ for FMB in both traction and braking, which may be due to the balance in the removal of the leaf layer and FMB from the disks surfaces. This optimum slip was observed with FMA only for braking; the moderate adhesion characteristic of FMA breaks the tendency for traction, as 
Fig. 9 Leaf contaminated tests at $2 \%$ slip (and reference baseline-dry without contamination)

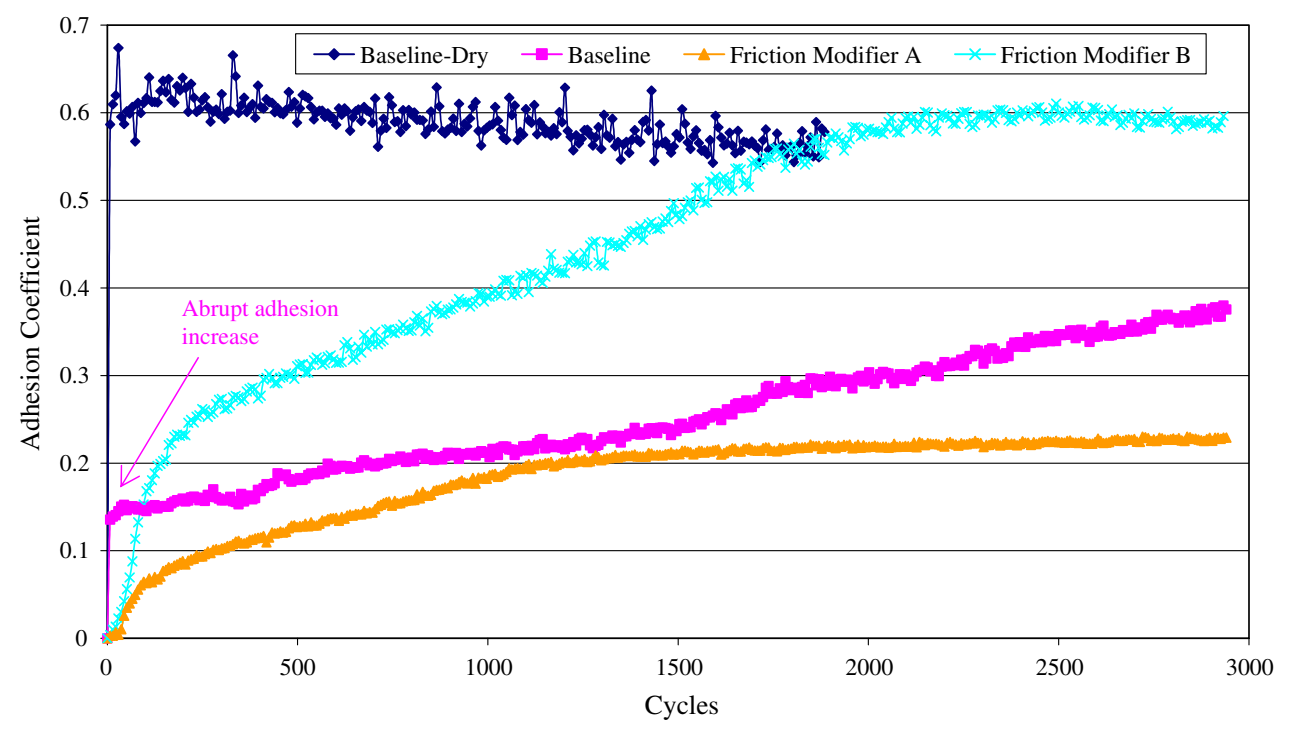

Table 1 Number of cycles required to reach the adhesion for adequate braking and traction performance

\begin{tabular}{llllllrr}
\hline & \multicolumn{3}{l}{ Braking $(\mu=0.09)$} & & & Traction $(\mu=0.2)$ & \\
\cline { 2 - 3 } \cline { 7 - 8 } & $\begin{array}{l}0.5 \% \\
\text { slip }\end{array}$ & $\begin{array}{l}1 \% \\
\text { slip }\end{array}$ & $\begin{array}{l}2 \% \\
\text { slip }\end{array}$ & & $\begin{array}{l}0.5 \% \\
\text { slip }\end{array}$ & $\begin{array}{l}1 \% \\
\text { slip }\end{array}$ & $\begin{array}{r}2 \% \\
\text { slip }\end{array}$ \\
\hline Baseline & 515 & 115 & 0 & 2255 & 982 & 667 \\
FMA & 245 & 207 & 220 & Not reached & 1884 & 1151 \\
FMB & 187 & 35 & 70 & & 1965 & 65 & 145 \\
\hline
\end{tabular}

shown in Table 1. In addition, for the baseline it was found that the higher the slip the better the performance, as it could be expected due to the associated higher rate of removal of the leaf layer.

\subsection{IR Spectroscopy Analysis}

After running each adhesion test, the remaining layers were analyzed using FTIR micro-spectroscopy. By looking at the organic components of those layers, it was possible to determine whether the leaf layer had been removed from the disk surface. In order to establish a reference, IR microreflection spectra of the sycamore leaf and the FMs were taken.

In Fig. 10 the spectra of the remaining layers in the adhesion tests with FMA are depicted; the leaf and FMA samples used in those tests are also included together with the leaf layer resulting from the preliminary feeding stage before FMA is applied. Similar spectra can be observed for the leaf sample and the created leaf layer. There was a broad reflection peak from 3100 to $3700 \mathrm{~cm}^{-1}$ that is presumably due to water $\mathrm{OH}$ stretch vibrations. The two characteristic peaks at 2920 and $2850 \mathrm{~cm}^{-1}$ could be related to lignin present in the leaf sample, while the absorption profile from 1800 to $800 \mathrm{~cm}^{-1}$ is from other compounds present in leaves thoroughly explained in [11]. It is worthwhile to mention that the peak at $1600 \mathrm{~cm}^{-1}$ could be attributed to lignin because the riblets of our leaf samples were not removed. Furthermore, FMA showed a high moisture content (peak at $3100-3700 \mathrm{~cm}^{-1}$ ), a characteristic peak at $1640 \mathrm{~cm}^{-1}$, and a rise in absorbance below $900 \mathrm{~cm}^{-1}$. Moreover, the spectra of the remaining layer in the test at $2 \%$ slip showed the most similar pattern with the leaf layer. It can also be seen that there was some FMA in that layer, which caused the rise in absorbance below $860 \mathrm{~cm}^{-1}$. Hence, FMA seemed to mix with the leaf layer in that test forming a layer that led to a reduction of $60 \%$ in the adhesion coefficient compared to the dry uncontaminated contact at $2 \%$ slip (see Fig. 9). Similar findings applied to the post-test layer at $1 \%$ slip, in which the reduction of the adhesion coefficient was $42 \%$.

FMB showed mainly the same characteristic peaks in spectra as FMA (see Fig. 11); however, different spectra peaks were observed in the range of 1500 to $1000 \mathrm{~cm}^{-1}$. In the tests with FMB, post-test layers were only found at $0.5 \%$ slip. This remaining layer seemed to have some FMB left as shown by the rise in absorbance below $820 \mathrm{~cm}^{-1}$. Some of the characteristic peaks of the leaf layer spectra were also observed, e.g. at wavelengths $1600 \mathrm{~cm}^{-1}$ and $1020 \mathrm{~cm}^{-1}$ on the post-test layer spectra, which would indicate that the leaf layer was not completely removed in the test. Thus, the adhesion did not fully recover with $30 \%$ decrease compared to the dry uncontaminated contact, as shown in Fig. 7.

\subsection{Leaf Layer and FMs Solid Particles Analysis}

Figure 12 (left) shows an example of the leaf layer generated after 4,000 cycles of run-in and 300-400 cycles of 
Fig. 10 IR micro-reflection spectra of initial samples, leaf layer, and post-test layers with FMA

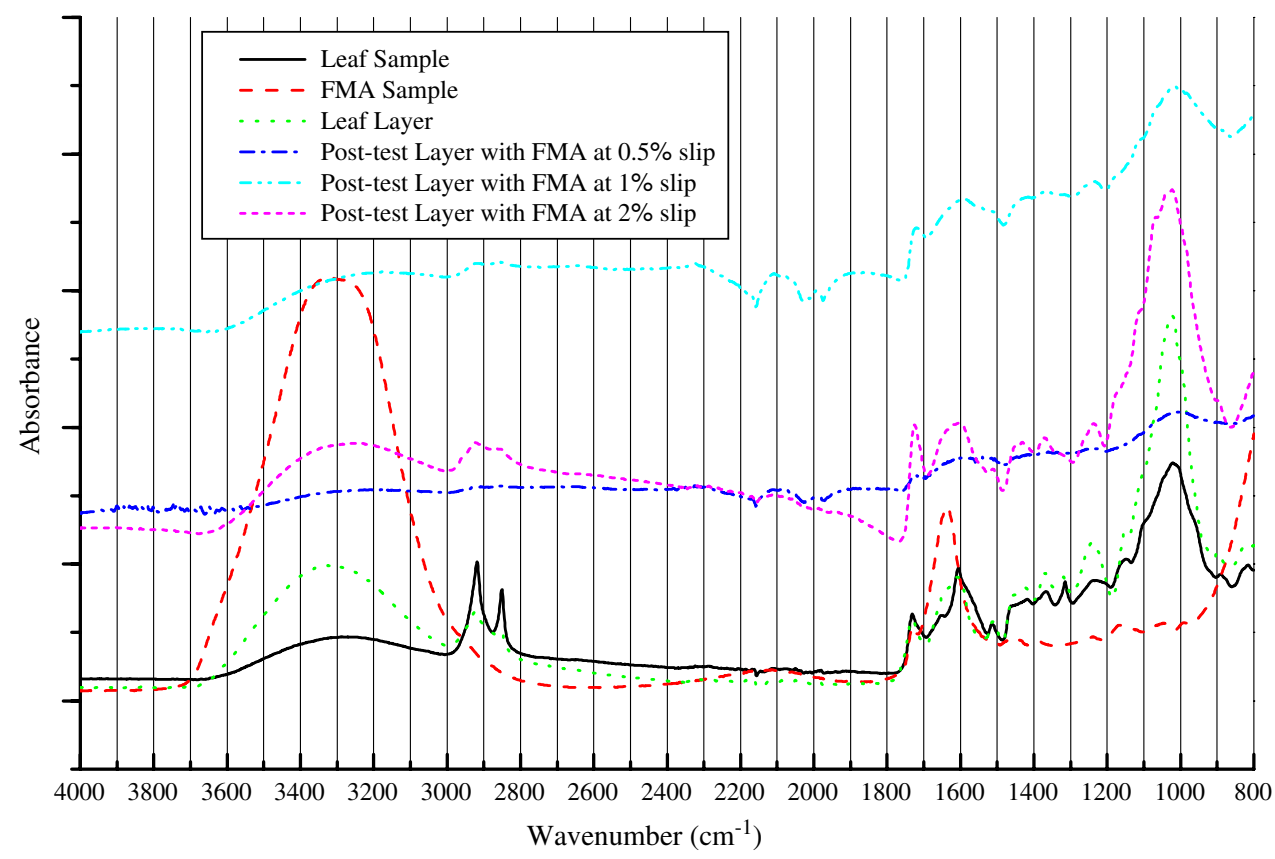

Fig. 11 IR micro-reflection spectra of initial samples, leaf layer, and post-test layer with FMB

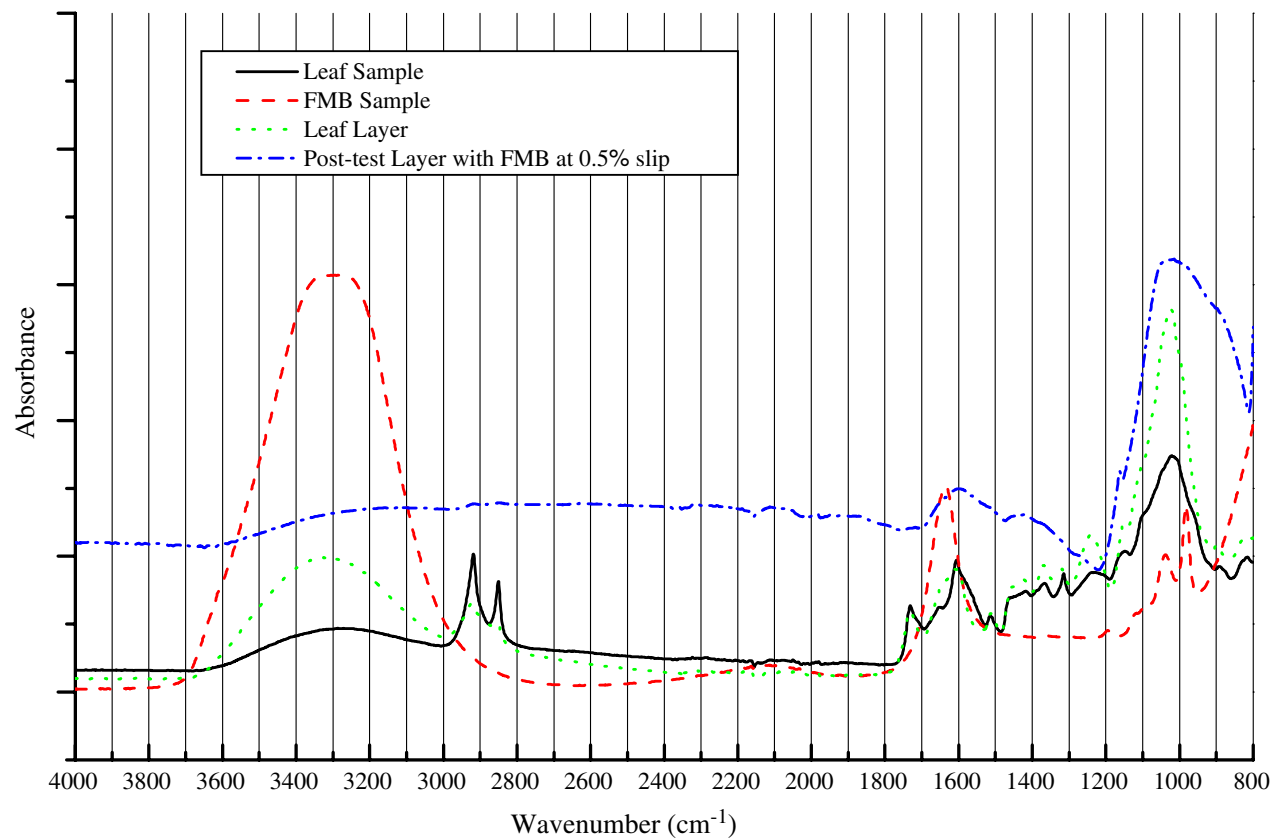

leaves application. Different coloration is observed on the layers present on the disks surface. Some light greenish layers correspond to adhered leaf mulch; whereas the majority of the layers present a black coloration and are firmly adhered on the disks surfaces. The latter layers have been identified in this paper as the black leaf layer that is equivalent to that found on the railway track. In Fig. 12 (right) the remaining layer on the disks surfaces after 3,000 cycles of removal test is shown. The previously generated black leaf layer is not fully removed from the disks surface after 3,000 cycles in baseline conditions at $0.5 \%$ slip, as some patches still adhere to the disk surface. This proves that the generated black leaf layer is very durable as it has also been reported from observations on the track $[5,6]$.

The particle size of the FMs determines whether the particles could prevail over the leaf layer to interact with wheel and rail in a metal-particle-metal contact, as compared to the thickness of the leaf layer. The particle size distribution of both FMs was measured by means of a laser particle analyzer; the results are depicted in Fig. 13. In FMA two size ranges of solid particles were predominant in the mix: 10 and $100 \mu \mathrm{m}$. The solid particles of FMB had 
Fig. 12 Leaf layers on the disks surface after generation test (left) and after removal test (right)
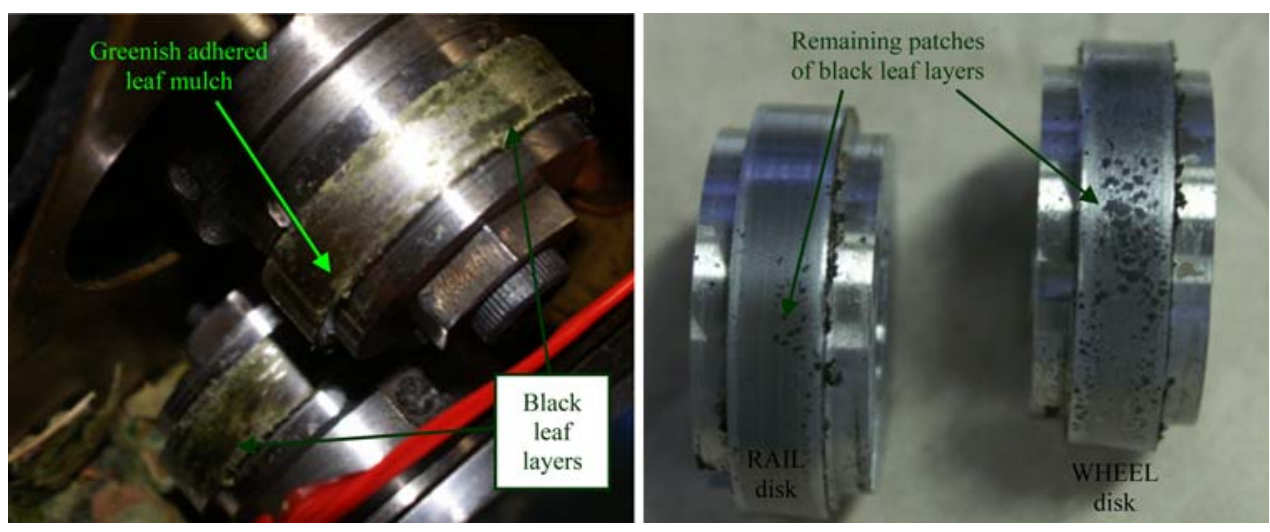

Fig. 13 Particle size distribution of FMA and FMB

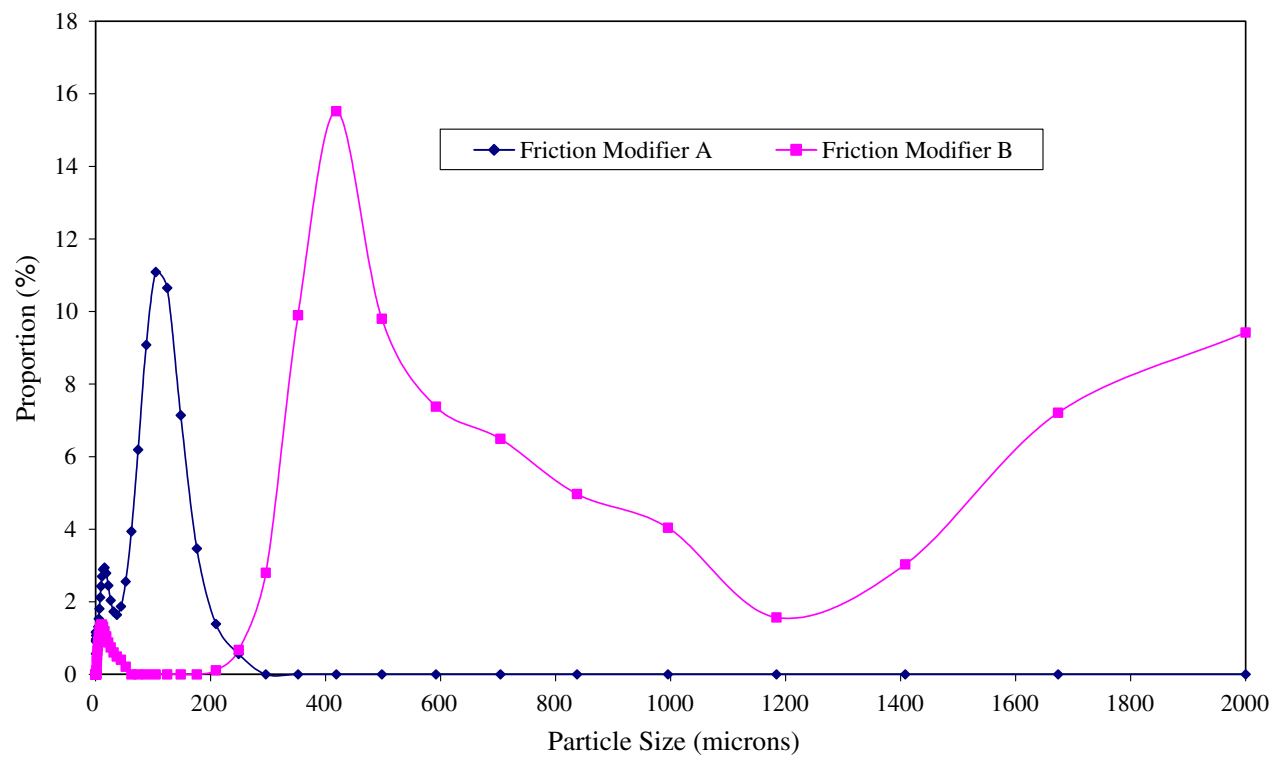

a larger size, which ranged from 300 to $2,000 \mu \mathrm{m}$. The thickness of the post-test leaf layer (i.e., after 3.000 cycles of removal test) was measured with an optical 3D profiling system WykoNT3300 (Veeco Metrology Group, USA). The values of thickness ranged 3-13 $\mu \mathrm{m}$.

In order to study the rupture strength and associated particle size change, particle strength analyses of the FMs were carried out by means of a high precision press in the laboratory. An individual particle of each FM was put between two metal plates and subject to normal load in a displacement controlled process. Figure 14 depicts the most representative tests. The difference in stiffness of the particles contained in FMB (i.e., stainless steel and sand) was observed in terms of different initial load-deflection slope. The sand particles had a steeper slope and presented a brittle behavior. They could be either crushed for a few times into dust (as shown with particle FMB_S2) or be embedded in the softer steel material (see particle FMB_S1). If the sand particles were crushed, smaller particles were formed that could bear the load until dust was finally formed. The reduction in size in the tests ranged from 50 to $400 \mu \mathrm{m}$, until no more load could be born by the particles. On the other hand, the stainless steel particles underwent plastic deformation owing to their ductility; some particles just flattened and remained unbroken throughout the test (as seen in FMB_SS2), some broke and were further deformed (as observed in FMB_SS1). There was a slight change in slope between FMB_SS1 and FMB_SS2, which can be attributed to the amorphous shape of the particles that leads to a different contact area. The solid particles contained in FMA broke up at smaller loads (around $0.5 \mathrm{~N}$ ) due to its small size compared to FMB. The decrease in size ranged from 20 to $80 \mu \mathrm{m}$ before dust was formed.

In addition, the hardness of the solid particles contained in the FMs will determine the effectiveness of cutting through the leaf layer. The hardness of the leaf layer remaining after each test was measured by means of Vickers micro-indentation technique; average values between $47 \mathrm{HV}_{10 \mathrm{~g}}$ and $68 \mathrm{HV}_{10 \mathrm{~g}}$ were obtained depending on the degree of compaction of the layer. This is in line with previous work carried out with leaves on the same 
Fig. 14 Particle strength tests of FMB ( $\mathrm{SS}=$ stainless steel, $\mathrm{S}=$ sand) and FMA solid particles

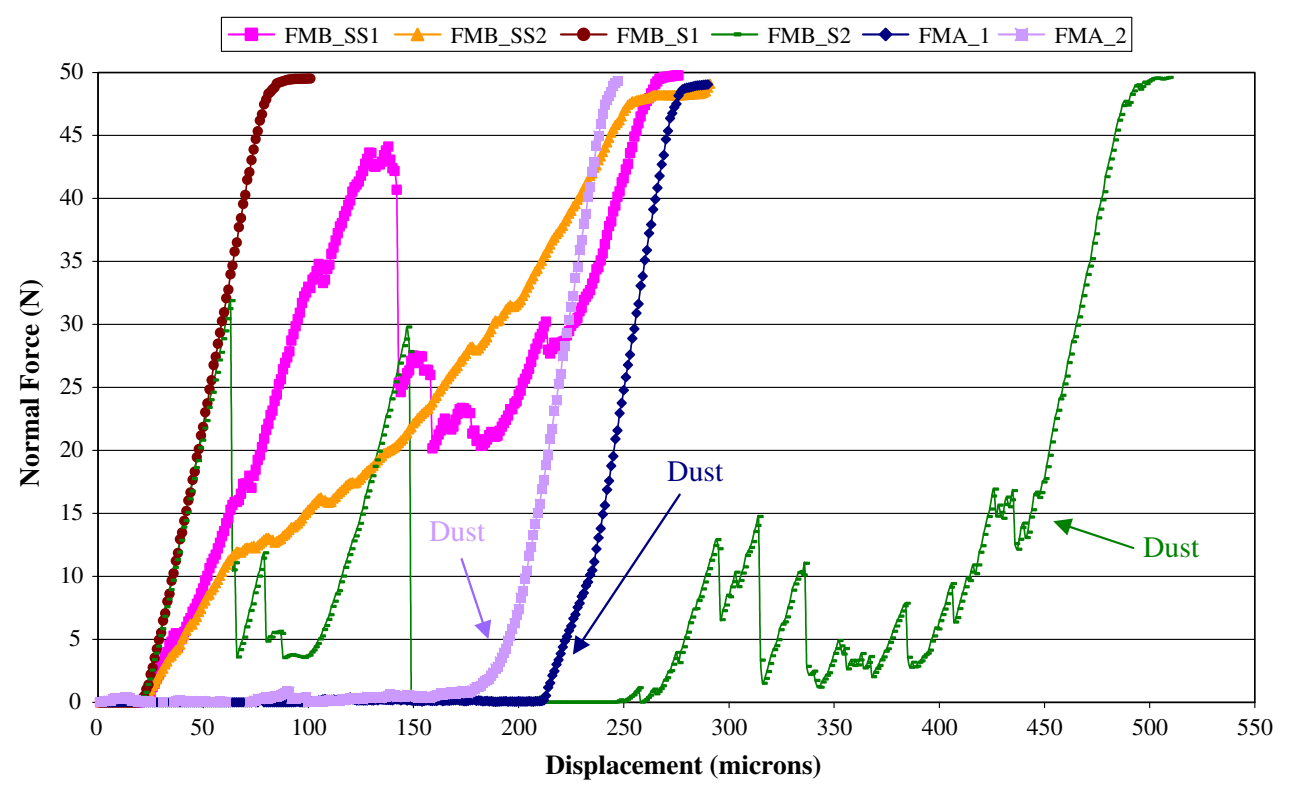

roller rig [12]. The hardness of the solid particles of both FMs was also measured using the same technique. The stainless steel particles of FMB gave an average 320 $\mathrm{HV}_{10 \mathrm{~g}}$, while an average of $1500 \mathrm{HV}_{10 \mathrm{~g}}$ was obtained for the sand particles. Despite many attempts, the hardness measurements of the particles contained in FMA were not successful due to their small size.

\subsection{Disk Surface Analysis}

After completion of the tests, the surface of the disks was examined. No surface damage was found in the baseline tests. In the tests with FMB, the hard solid particles, which were responsible for cutting through the leaf layer, caused indentations on the surface of both wheel and rail disks. The indentations varied in size from 0.8 to $2 \mathrm{~mm}$ in equivalent diameter and with $50 \mu \mathrm{m}$ depth on average. A picture of an indented rail disk together with a microphotograph of the indentation is given in Fig. 15. Similar findings for different solid contaminants have been presented by other researchers [13, 18], and also in a previous work carried out with FMB in dry and wet contacts [16]. On the contrary, no surface damage was observed when using FMA.

\section{Discussion}

The adhesion coefficient during the feed of leaves ranged from 0.01 and 0.04 for $0.5 \%$ slip. Once the feed of leaves was stopped, the adhesion increased with the cycles as the leaf layer was removed from the disks surfaces. FMB showed the fastest recovery in adhesion for both braking and traction. It was up to $70 \%$ faster in braking and up to 93\% faster in traction compared to the baseline. Hence, when using FMB in real wheel-rail systems the number of wheel passages needed to restore adhesion to an adequate level for traction could be reduced by a factor of up to 15 ; whereas in braking the improvement factor could reach up to 3. However, these results can only be taken as a qualitative indication of the actual wheel-rail situation because of the differences with the twin-disk setup, as already
Fig. 15 a Picture of an indented rail disk, $\mathbf{b}$ micrograph of an indentation
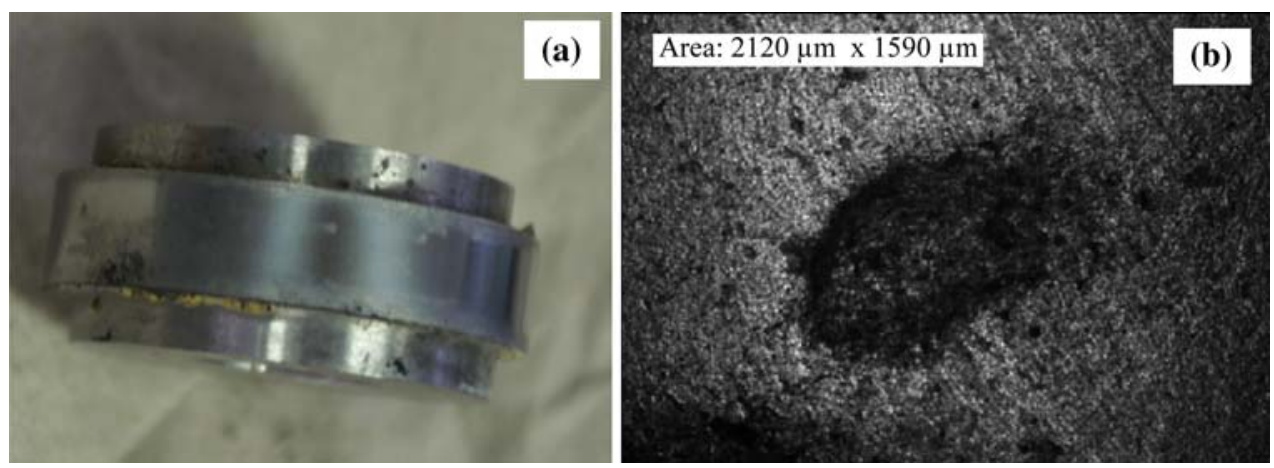
outlined in [15]. On the other hand, the moderate adhesion characteristics of FMA brought about slower recoveries in adhesion for traction requirements when compared to the baseline for all slips considered. In addition, it is worth to notice that in this work we have tested the traction operation, in which the wheel slips over the rail; however, we have also used our results for comparisons with the braking requirements. In braking operation, the rail would be represented by the faster disk and the wheel by the slower one. Nevertheless, previous research has shown that there exists a negligible influence on the adhesion results when changing the direction of the slip [19]; therefore, our adhesion results can be used for both traction and braking.

By means of these tests, the optimum slip ratio for the best adhesion recovery in the different contact conditions could be investigated. For the baseline, it was found that faster adhesion recovery was achieved at higher slips, as it could be expected due to the increased removal effect. An interesting direction of research would be to study which optimum slip is necessary to remove the leaf layer without much additional damage to wheels and rails. If train-borne leaf layer detection techniques were developed, the wheels could be set to a certain slip in order to remove the leaf layer from the rails for the subsequent wheels. In the tests with FMB, an optimum was observed at $1 \%$ slip in reaching both braking and traction adhesion requirements. This optimum can be explained as a balance between the amount of FMB and leaf layer that is removed from the disks surface. When using FMA, the optimum could also be observed at $1 \%$ slip, but only from the point of view of braking. For traction, it seems that FMA cannot bring the adhesion to what is required, unless FMA is removed from the disks surface. This finding is in good agreement with observations from previous work with FMA in dry contacts, in which it was found that FMA decreased adhesion compared to the baseline to moderate values in uncontaminated dry conditions [16].

When compared to the thickness and hardness of the leaf layer, the size and hardness of the solid particles contained in the FM will primarily determine the capacity to break up the leaf layer and restore the adhesion back to acceptable levels. Measurements of the leaf layer thickness in both field and laboratory scale have been performed in the United Kingdom [5, 10]. The thickness ranged from 10 to $100 \mu \mathrm{m}$ depending on the compaction to which the leaves had been subjected. Samples of leaf layer taken from the Dutch railways network in autumn 2006 have been measured with thickness 20-30 $\mu \mathrm{m}$. In our laboratory tests, the thickness of the post-test leaf layer ranged 3-13 $\mu \mathrm{m}$. This layer thickness is larger than the wheel and rail disks roughness; therefore, the layer may inhibit the metal-metal contact. The particle size of the FM determines whether the solid particles will interact with the wheel and the rail in a metal-particle-metal contact. The solid particles of FMB could prevail over the leaf layer due to its large size, while the initial particle size of FMA is in the same range as the leaf layer thickness. Nevertheless, one must bear in mind that the majority of the particles will break up due to the high wheel-rail contact pressure. This phenomenon was investigated for sand particles [13] and for crushed granite ballast [18]. Broken-up particles will have smaller size and may be entrapped within the third-body leaf layer; thus, losing its effectiveness. The strength analysis tests showed a reduction of $20-80 \mu \mathrm{m}$ in size for the solid particles of FMA and 50-400 $\mu \mathrm{m}$ for the sand particles contained in FMB. The stainless steel particles of FMB either deformed due to their ductility or broke with a maximum reduction in size of $200 \mu \mathrm{m}$. Hence, the brokenup FMA particles may be entrapped within the third-body leaf layer, whereas the solid particles of FMB would still have larger dimensions than the leaf layer thickness. Moreover, considerations on the feasibility of small particles to get entrained in the wheel-rail contact must be taken into account, as it was already mentioned in [13]. Observations during the testing showed that a great number of particles of FMB were expelled due to their large particle size. Therefore, the size of the solid particles of a FM should be optimized toward adequate particle entrapment and efficacy against leaf layer thickness.

In addition, the hardness of the solid particles of the FM will determine the effectiveness of cutting through the leaf layer. The hardness of the leaf layer mainly depends on the degree of compaction (given by the contact load and wheel slip) and the water content. It was shown in previous laboratory tests that dry leaf layers are harder than wet ones [12]. In fact, the softening effect of water can help in the removal of the leaf layer, as already mentioned in the literature $[5,10]$. In this paper, only dry leaf layers have been tested, as they represent the hardest to be removed from the wheel and rail surfaces. In field and full-scale tests carried out in The United Kingdom, leaf layers have been reported to have a hardness of 1-4 in Mohs scale [10]. In previous work with dry and wet leaves on the SUROS roller rig, micro-hardness of the layer was reported to range from 15 to $60 \mathrm{HV}_{1 \mathrm{~g}}$ [12]. In this paper, the micro-hardness of the layers ranged 47-68 $\mathrm{HV}_{10 \mathrm{~g}}$ depending on the degree of compaction of the layer. The solid particles of FMB are harder than the leaf layer. Accordingly, the solid particles could effectively cut through the leaf layer, leading to a fast recovery in adhesion as shown in this paper. Conversely, they caused indentations on the surface of both wheel and rail disks. FMA did not show as effective break-up of the leaf layer as FMB; however, no indentations were observed in the tests with FMA. Hence, it can be concluded that the hardness of the solid particles of a FM should be optimized to a compromise between effective leaf layer removal and minimized surface damage to wheel and rail. 
Moreover, it is worthwhile to emphasize that the study here presented corresponds to the post-application of the FMs, i.e., application of the FM once the leaf layer is already present on the disks surfaces. An interesting possibility and still open question is whether the pre-application of the FM would be effective in hindering the formation of the leaf layer. Some trials were carried out by the authors, in which the rail disk was coated with FM before leaves were applied. Unfortunately, those trials did not lead to clear results due to stability problems of the roller rig that yielded wrong measurements in the torque transducer.

\section{Conclusions}

The leaf contaminated wheel-rail contact has been simulated in rolling-sliding conditions with a twin-disk roller rig in closely controlled laboratory tests. The leaf layer has been generated in similar conditions to the actual wheelrail contact. Two water-based FMs have been tested in order to evaluate their performance to overcome low adhesion problems associated with leaves. FMB has been extensively used in the Dutch and British railways networks for the last years to overcome adhesion problems, especially leaf-related ones during autumn. FMA was tested successfully in wet contacts and its performance in leafy contacts was of much interest. In order to compare the results of the FMs with the untreated conditions (i.e., without FM), a baseline has also been tested. This work yields the following conclusions:

(a) In the presence of leaf layer the adhesion coefficient is between 0.01 and 0.04 . As the leaf layer is removed after some necessary cycles, adhesion recovers to a certain degree. FMB shows the largest adhesion recovery as its large hard solid particles effectively break up the leaf layer.

(b) FMB leads to the fastest adhesion improvement in both traction and braking requirements with a reduction in cycles of up to 93 and $70 \%$, respectively, when compared to the baseline. FMA seems to be slower than the baseline due to its moderate adhesion characteristics.

(c) An optimum in adhesion recovery is found at $1 \%$ slip for FMB due to the balance between leaf layer and FMB removal. For FMA the optimum is also at $1 \%$ for braking, while for traction the higher the slip the better the performance due to its moderate adhesion characteristics. In baseline conditions, higher slip leads to better performance in both traction and braking because of the increased removal effect.

(d) FTIR micro-spectroscopy showed that FMA seems to mix up with the leaf layer forming a layer that reduces the adhesion coefficient between 42 and $60 \%$ in the tests with FMA at 1 and $2 \%$ slip, respectively, as compared to the clean dry conditions.

(e) Two parameters of an FM play the main role in adhesion recovery: the hardness of the solid particles and the particle size. These two parameters need to be designed in accordance with the hardness and thickness of the leaf layer so as to optimize the removal of leaf layer.

(f) The large hard particles of FMB cause indentations to the wheel and rail disk surfaces; whereas, no indentations are observed as caused by the small particles of FMA. Therefore, a compromise needs to be found for the hardness and size of the solid particles when a FM is developed in order to cut through the leaf layer and not cause severe surface damage on wheel and rail.

Acknowledgments The authors gratefully acknowledge Dr Mike Frolish from the Engineering Materials Department of The University of Sheffield for his support in conducting the tests.

Open Access This article is distributed under the terms of the Creative Commons Attribution Noncommercial License which permits any noncommercial use, distribution, and reproduction in any medium, provided the original author(s) and source are credited.

\section{References}

1. Beagley, T.M., McEwen, I.J., Pritchard, C.: Wheel-rail adhesion boundary lubrication by oily fluids. Wear 31, 77-88 (1975). doi: 10.1016/0043-1648(75)90123-4

2. Beagley, T.M., McEwen, I.J., Pritchard, C.: Wheel/rail adhesion - the influence of railhead debris. Wear 33, 141-152 (1975). doi:10.1016/0043-1648(75)90230-6

3. Beagley, T.M., Pritchard, C.: Wheel/rail adhesion-the overriding influence of water. Wear 35, 299-313 (1975). doi:10.1016/ 0043-1648(75)90078-2

4. Beagley, T.M.: The rheological properties of solid rail contaminants and their effect on wheel/rail adhesion. Proc. Inst. Mech. Eng. 190, 419-428 (1976). doi:10.1243/PIME_PROC_1976_ 190_044_02

5. Fulford, C.R.: Engineering Review of Low Adhesion Research. Report published by Rail Safety and Standards Board, UK (2004)

6. Adhesion orking Group: Managing Low Adhesion. Report published by the Adhesion Working Group, UK (September 2001)

7. Li, Z., Zhao, X., Esveld, C., Dollevoet, R., Molodova, M.: An investigation into the causes of squats-correlation analysis and numerical modeling. Wear 265, 1349-1355 (2008)

8. Olofsson, U., Sundvall, K.: Influence of leaf, humidity and applied lubrication on friction in the wheel-rail contact: pin-ondisc experiments. J. Rail. Rapid. Transit. 218, 235-242 (2004). doi:10.1243/0954409042389364

9. Press release by the Dutch Railways in Dutch, www.ns.nl (October/November 2002)

10. Poole, W.: Characteristics of Railhead Leaf Contamination. Summary Report published by Rail Safety and Standards Board (May 2007)

11. Cann, P.M.: The 'leaves on the line'-a study of leaf residue film formation and lubricity under laboratory test conditions. Tribol. Lett. 24, 151-158 (2006). doi:10.1007/s11249-006-9152-2 
12. Gallardo-Hernandez, E.A., Lewis, R.: Twin disk assessment of wheel/rail adhesion. Wear 265, 1309-1316 (2008)

13. Lewis, R., Dwyer-Joyce, R.S.: Wear at the wheel/rail interface when sanding is used to increase adhesion. J. Rail. Rapid. Transit. 220, 29-41 (2006). doi:10.1243/095440905X33260

14. Eleveld, J.: Gladde Sporen, Oplossingsrichtingen voor Adhesieproblemen. Report published by the Dutch Railways Technical Research (NSTO) in Dutch (March 1999)

15. Olofsson, U., Lewis, R.: Tribology of the wheel-rail contact. In: Iwnicki, S. (ed.) Handbook of Railway Vehicle Dynamics, pp. 121-141. Taylor \& Francis Group, Boca Raton (2006)

16. Arias-Cuevas, O., Li, Z., Lewis, R., Gallardo-Hernandez, E.A.: Rolling-Sliding Laboratory Tests of Friction Modifiers in Dry and Wet Wheel-Rail Contacts to be published
17. Fletcher, D.I., Beynon, J.H.: Development of a machine for consely controlled rolling contact fatigue and wear testing. J. Test. Eval. 28, 267-275 (2000)

18. Grieve, D.G., Dwyer-Joyce, R.S., Beynon, J.H.: Abrasive wear of railway track by solid contaminants. J. Rail. Rapid. Transit. 215, 193-205 (2001). doi:10.1243/0954409011531512

19. Deters, L., Proksch, M.: Friction and wear testing of rail and wheel material. Wear 258, 981-991 (2005). doi:10.1016/j. wear.2004.03.045 